\title{
Education and Outreach Activities in Astroparticle Physics offered by Netzwerk Teilchenwelt
}

\author{
Hans-Peter Bretz* \\ DESY, Platanenallee 6, 15738 Zeuthen, Germany \\ E-mail: hans-peter.bretz@desy.de
}

\begin{abstract}
What are cosmic particles and where do they come from? These are questions which are not only fascinating for scientists. The German education and outreach organization "Netzwerk Teilchenwelt" offers a large variety of projects and activities to bring the methods and newest results of high energy and astroparticle physics to high school students and teachers. Several experiments with a focus on the latter have been established which can be built and conducted in the classroom. Cloud chambers help students understand basic methods of detecting charged particles. Scintillation detectors as well as small water Cherenkov detectors based on coffee thermos and photomultipliers can be used to measure the flux of secondary particles from cosmic ray induced air showers. These setups enable the students to do their own measurements, e.g. the zenith angle distribution of the flux or the half life of muons. Additionally students can participate in masterclasses either at schools or organized as international masterclasses, where they can analyze real data from the Pierre Auger Observatory or the IceCube Neutrino Observatory.
\end{abstract}

The European Physical Society Conference on High Energy Physics

22-29 July 2015

Vienna, Austria

${ }^{*}$ Speaker, on behalf of Netzwerk Teilchenwelt. 


\section{Introduction}

The German organization "Netzwerk Teilchenwelt" (engl.: network particle world) is an education and outreach network that focuses on particle and astroparticle physics. Its aim is to engage high school students in modern physics by letting them experience research, get in contact with scientists and conduct their own experiments. The network consists of 26 German universities and research institutes with the Dresden University of Technology as project leader and additionally CERN as partner for organizing workshops. The activities related to astroparticle physics are coordinated by DESY in Zeuthen. The partnering institutions can offer activities for students in particle physics and astroparticle physics, international masterclasses as well as training for teachers. All activities are usually led and organized by science students and young scientists who are active in the respective fields. Figure 1 shows a map of all sites that offer astroparticle physics as part of their outreach program. This article gives an overview of the activities related to astroparticle physics. Section 2 describes experiments that can be conducted in the classroom by students and teachers together. Sections 3 and 4 comprise day-filling programs offered to students at their schools or at the partnering institutions that combine introductions into the basic science as well as experiments or data analysis conducted by the students.

\section{Experiments}

\subsection{Measuring Cosmic Ray Particles}

Netzwerk Teilchenwelt offers students the opportunity to conduct experiments on their own

1. University of
Kiel
2. University of
Rostock
3. DESY Hamburg
4. DESY Zeuthen
5. University of
Münster
6. University of
Göttingen
7. University of
Duisburg-Essen
8. University of
Wuppertal
9. Dresden
University of
Technology
10. RWTH Aachen
University of
Technology

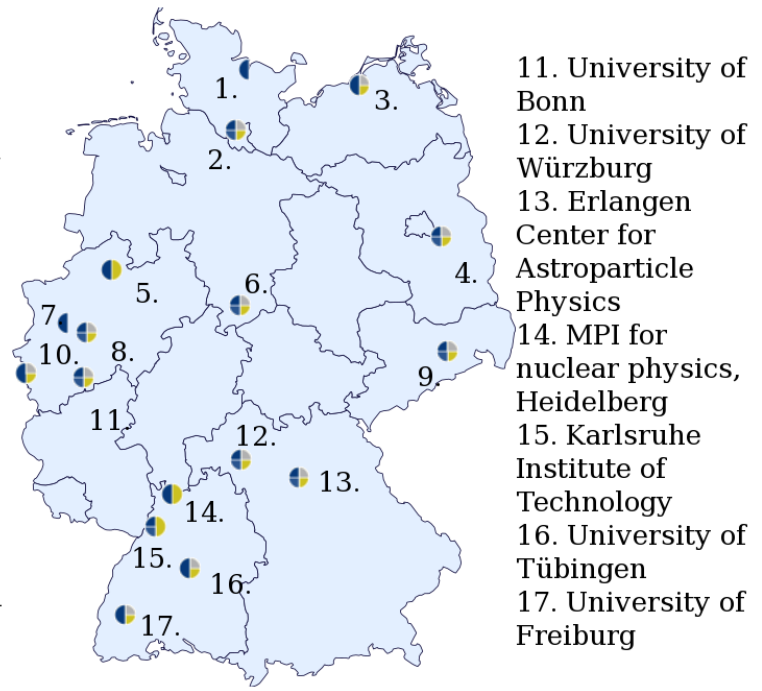

Figure 1: Overview of the 17 German institutions that are part of Netzwerk Teilchenwelt and offer activities in the field of astroparticle physics. The colors in the circle indicate the other activities offered at the same location. Yellow stands for particle physics, grey for international masterclasses and blue for training programs aimed at teachers and project leaders. Additional workshops as well as seminars for teachers are hosted and coordinated at CERN. 


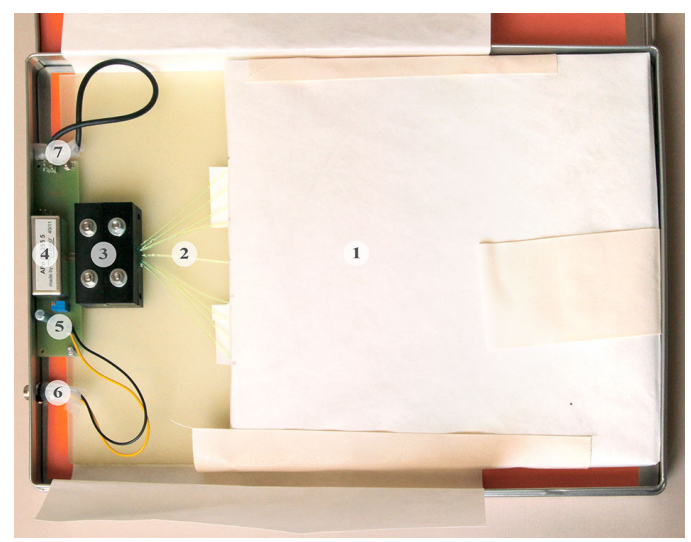

(a) Detailed view of a scintillator box.

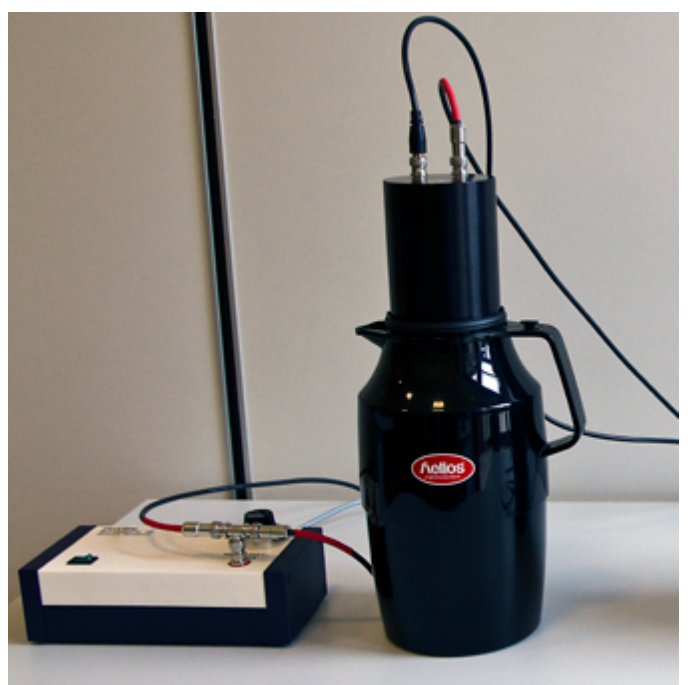

(b) Water Cherenkov detector with a coffee thermos as detector volume.

Figure 2: Figure 2a shows an open scintillator box: 1. scintillator plate wrapped in paper, 2. optical fibers attached to the MMPC (3), 4. voltage converter, 5. resistor for voltage adjustment, 6. connector for power supply, 7. analogue output for MPPC signal. From [1]. Figure 2b shows a small water Cherenkov detector consisting of a high voltage supply, a photomultiplier tube and a coffee thermos.

and follow up on their individual ideas. The experimental setups can be used in outreach projects at research institutes or directly at high schools. The institutions of Netzwerk Teilchenwelt offer training for teachers and lend the the setups to high schools. The hardware is designed to be robust and easily transportable.

\section{Detector Setup}

The Cosmic Muon Observer Experiment for Students (CosMO) [1] is a scintillation counter experiment based around the data acquisition (DAQ) card developed for QuarkNet [2]. It combines affordable components with a graphical user interface that implements different experiments on atmospheric muons.

The detector consists of three plastic scintillator units. Each scintillator consists a tile of $20 \times$ $20 \times 1.2 \mathrm{~cm}^{3}$ that is attached to a multi-pixel photon counter via nine optical fibers. The scintillator material and the MPPC are housed in a lightproof aluminum box with connectors for the voltage supply and coaxial cable for the analogue MPPC output signal. Figure $2 \mathrm{a}$ shows the inside of a scintillator unit. Figure $2 b$ shows an alternative detector. It consists of a coffee thermos that is filled with distilled water. A photomultiplier tube is attached to the thermos in place of the lid and submerged in the water. A relativistic particle traveling through the volume of the thermos will create Cherenkov light that is reflected at the inner glass walls and creates a pulse in the photomultiplier. Both setups can be connected to a QuarkNet DAQ card and the data subsequently 


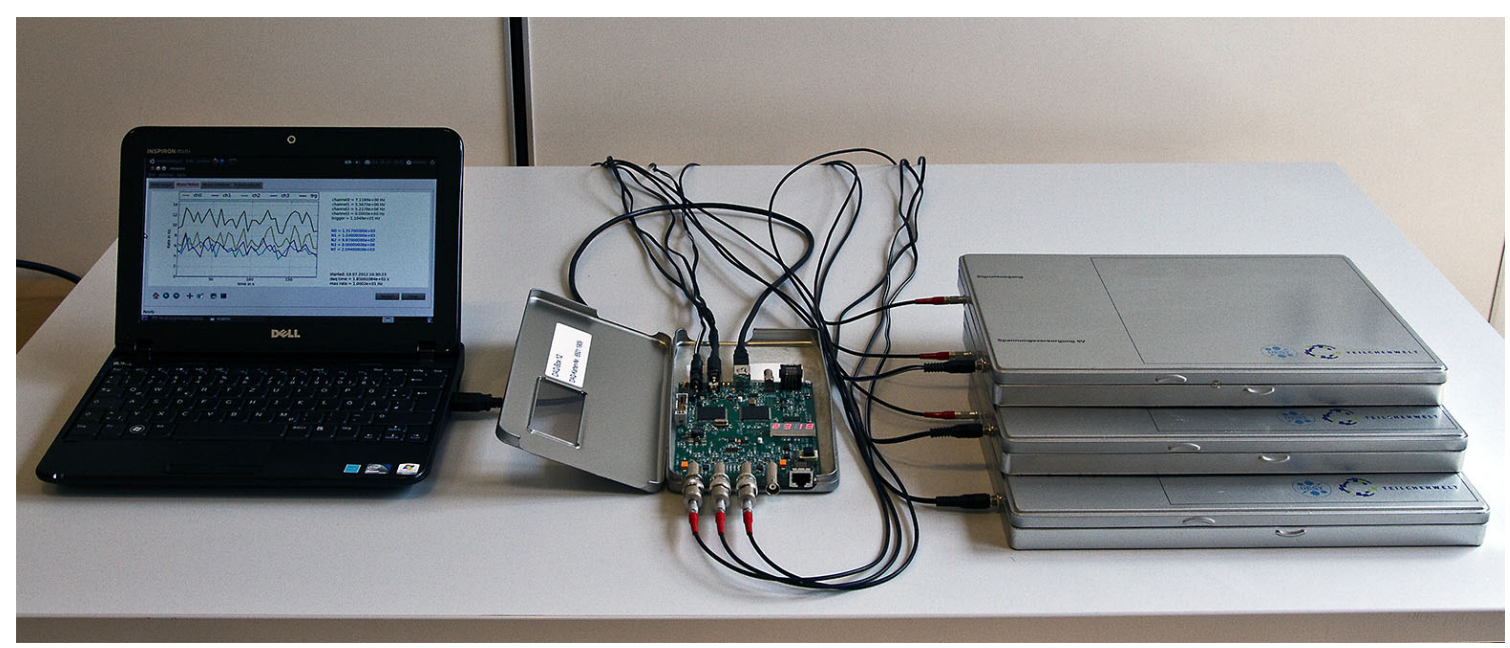

Figure 3: Overview of the experimental setup of CosMO. Three scintillator plates (right) are attached to a DAQ card (center). The laptop on the left-hand side shows the graphical user interface with an ongoing muon rate measurement. From [1].

be analyzed with the same software.

\section{User Interface}

In order to enable all students to analyze data independent of their programming background, the easy-to-use graphical user interface muonic has been created. It has been developed entirely in Python and is available as open source code at [3] as well as through the Python Package Index [4]. The user interface is divided into widgets for communication with the DAQ card as well analysis widgets for the individual experimental setups. The analysis widgets offer a live view of the respective data together with an evaluation of the results.

The rate widget shows the current event rates at the individual channels. A multiplicity trigger can be defined as well and be displayed together with the rates. This widget can be used e.g. to calibrate the voltage thresholds or to measure rates for different experimental setups. The velocity widget histograms the time difference between the signals at two detectors and can be used to determine the speed of the muons passing through. With three detector attached to the DAQ card, the muon decay widget can be used to determine the average life time of muons.

The combination of muonic and the DAQ card can be used to analyze any data from compatible photomultipliers.

\subsection{Cloud Chambers}

Cloud chambers represent an experimental setup that can easily be realized in a classroom with cheap material. Figure 4 shows a sketch of the setup that is distributed on request to high schools by Netzwerk Teilchenwelt. The chamber itself is a plastic box open on one side that rests on a metal plate. The metal plate lies in a Styrofoam padded tray filled with dry ice that provides the cooling for the cloud chamber. On the top of the plastic box is a piece of felt saturated with alcohol fastened to the inside of the box with small magnets. 


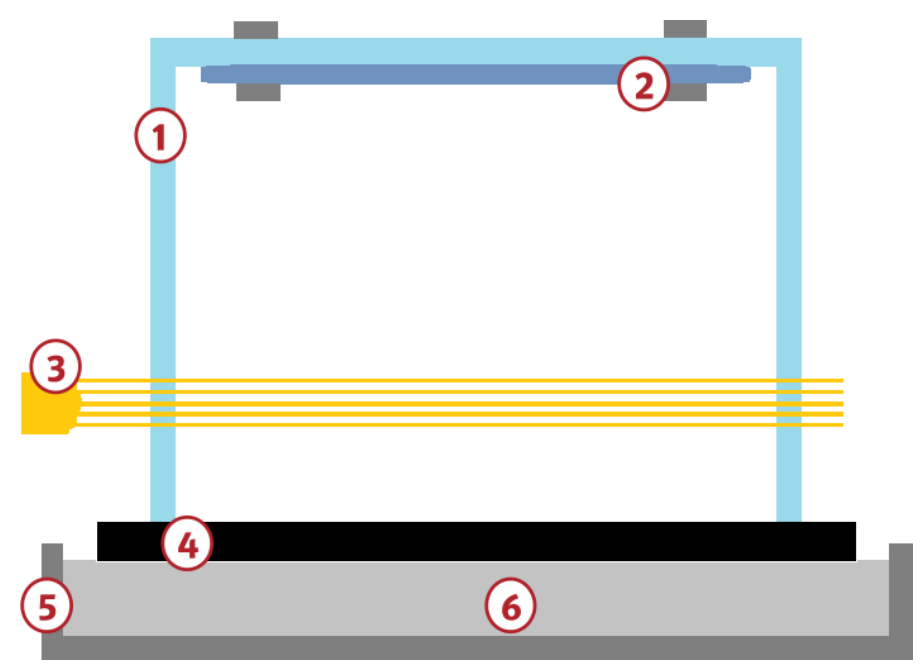

Figure 4: Schematic view of a cloud chamber: A felt soaked in alcohol (2) is attached via magnets to the top of a small plastic box (1) that stands on top of a metal plate (4). The plate lies in a Styrofoam coated box (5) filled with dry ice (6). A flash light is used to shine through the bottom (3) where the condensation along the tracks of charged particles occurs.

The cooling on the bottom of the chamber creates a temperature gradient inside the cloud chamber and thus a zone oversaturated with alcohol above the metal plate. Charged particles passing through this zone will act as condensation seed and create different traces of condensed alcohol depending on the type of charged particle.

The experiment can be done as part of another program, e.g. a masterclass, or as part of the regular curriculum. A list of materials can be found at [5] (German).

\section{Auger Masterclass}

The Auger Masterclass is a one-day program developed in Netzwerk Teilchenwelt [6] based on the example of the International Masterclasses in particle physics. It offers students the possibility to analyze real experimental data with simple methods available in every high school computer lab and with high school level mathematical methods.

The Pierre Auger Observatory is currently the biggest detector for ultra-high-energy cosmic rays (UHECRs) with an instrumented area of $3000 \mathrm{~km}^{2}$. It is located in Southern Argentina and comprises 1600 surface detector units (SD) and a total of 24 fluorescence telescopes located at four sites on the edges of the SD array. The SD units are water Cherenkov tanks that measure the light created by muons from cosmic ray induced extensive air showers passing through them. The coincident data taken at different SD tanks is then combined to reconstruct the properties of the air shower and the original primary cosmic ray particle.

The Pierre Auger Collaboration has made $1 \%$ of all data taken by the surface array publicly available through an online event display [7]. The events can be examined online or downloaded individually. Additionally a summary of all events can be downloaded in one file. All files are available as simple text files that can be processed in any spreadsheet software. In order to get 


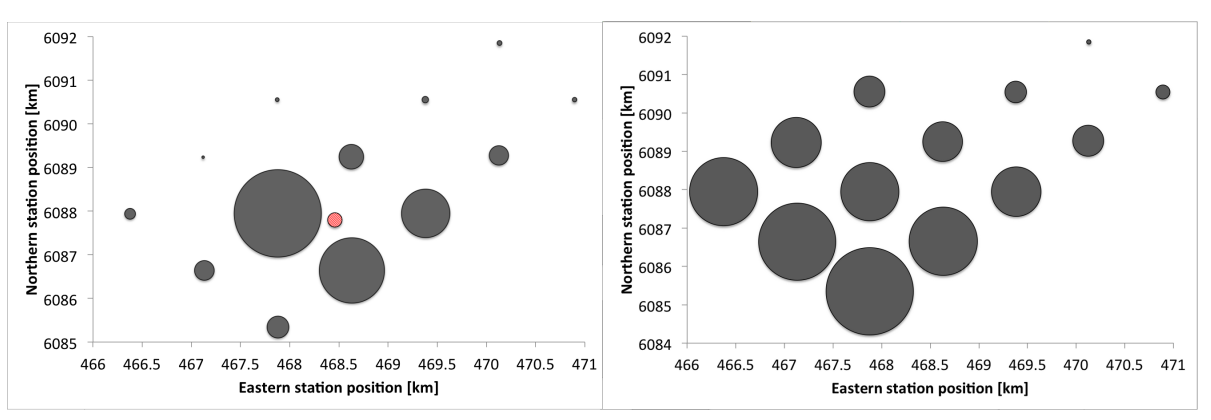

(a) Position and deposited energy of surface de-(b) Position and relative timing of the signals tectors.

measured in the surface detectors.

Figure 5: Two visualizations of an event created from data from the online event display. Figure 5a shows a bubble chart with the positions of the surface detectors and the signals they measured as the size of the dots. Figure $5 \mathrm{~b}$ shows the same event with the relative time delay as size of the dots. Both figures are taken from [6].

familiar with the data, students can start by reproducing typical event views as shown on the online event display with the means available through their locally installed software. Figure 5a shows a bubble chart showing the positions of the surface detectors that measured a signal for a certain event. The sizes of the data points indicate the charge measured at that location. In order to estimate the position of the shower axis, students can calculate the weighted mean of the detector positions weighted with the signal measured at that position which gives a good approximation of the shower axis as reconstructed by Auger.

In a similar fashion, students can visualize the time delay of the signal at the individual surface detectors in order to approximate the azimuth direction of the shower (figure 5b). With a given azimuth, students can try to derive a formula to estimate the zenith angle of the air shower. A detailed description of all steps can be found at [6].

\section{ICD}

The International Cosmic Day (ICD) is a yearly event first organized in 2012 by DESY in cooperation with Netzwerk Teilchenwelt in Germany and Fermilab with QuarkNet in the USA to celebrate the 100 year anniversary of the discovery of cosmic rays by Victor Hess [8]. During this day, high schools and universities worldwide are invited to participate and organize a program for high school students including introductions to cosmic ray physics and experiments conducted by the students. A live chat enables the student to exchange experiences and ideas. They can present their results at the end of the day in a video conference and summarize them on a page for the final result booklet. The high schools typically participate in cooperation with a nearby university or research institute which provides the experimental setup, e.g. scintillation detectors like the CosMO project. In 2015, over 20 institutions from 11 countries participated with a total of more than 400 students. 


\section{Summary}

Netzwerk Teilchenwelt offers a wide range of activities to promote astroparticle physics among high school students. All programs are organized in cooperation between research institutions and high schools. In programs like the International Cosmic Day or the Auger Masterclass students can participate and learn about the field of astroparticle physics. Analyzing data in a masterclass or conducting their own experiments with CosMO gives them an insight into modern research methods. After having taken part in a masterclass, the students can choose to support a scientist in teaching another masterclass on the same topic. Thus, students can experience all aspects of the work of a scientist.

\section{References}

[1] R. Franke, M. Holler, B. Kaminsky, T. Karg, H. Prokoph, A. Schönwald, C. Schwerdt, A. Stöß1, M. Walter, CosMO - A Cosmic Muon Observer Experiment for Students, Braz.J.Phys. 44 (2014) pp.415-608, [arXiv:1309.3391].

[2] S. Hansen, T. Jordan, T. Kiper et al., Low-cost data acquisition card for school-network cosmic ray detectors , IEEE. Nuclear Science Symposium Conference Record 1 (2003) 130-133

[3] https://github.com/achim1/muonic/

[4] https://pypi.python.org/pypi/muonic

[5] http://www.teilchenwelt.de/material/materialien-fuer-lehrkraefte/selbstbau-einer-nebelkammer/

[6] M. Krause, H. P. Bretz, L. Classen, M. Holler, M. Hütten, S. Raab, J. Rautenberg and A. Schulz, PoS ICRC 2015 (2015) 304, [arXiv:1508.03968].

[7] http://auger.colostate.edu/ED/

[8] http://icd.desy.de/ 